\title{
Nouveau protocole robuste pour les communications dans l'loV
}

\section{New robust protocol for loV communications}

\author{
Lylia Alouache ${ }^{1,2}$, Nga Nguyen ${ }^{1}$, Makhlouf Aliouat ${ }^{2}$, Rachid Chelouah $^{1}$ \\ ${ }^{1}$ Laboratoire Quartz, COMUE Paris Seine, EISTI, France, lae@eisti.eu,nn@eisti.eu,rc@eisti.eu \\ ${ }^{2}$ Laboratoire Réseaux et Systèmes Distribuées, Université Sétif, Algérie, aliouat_m@yahoo.fr
}

RÉSUMÉ. L'article présente d'abord quelques définitions sur les réseaux véhiculaires, les domaines d'application, les technologies de communication et les obstacles liés à la qualité de service (QoS) des communications de l'Internet des véhicules (loV). Ensuite, il introduit notre proposition sur la détection et le contournement des zones de déconnexions grâce à un nouveau protocole de routage géographique qui se base sur : i) l'estimation des durées de contact entre les véhicules, ii) les charges de données à transmettre et iii) les logs d'anomalies de communication. L'objectif est d'assurer la disponibilité, la fiabilité et la robustesse des communications intervéhiculaires en prenant en compte ces trois différents critères dans un algorithme d'acheminement des paquets de données.

ABSTRACT. First, the paper presents some definitions of vehicle networks, application areas, communication technologies and barriers that disrupt communications and deteriorate quality of service (QoS) in the Internet of Vehicles (IoV). Then, it introduces our proposition aiming to detect and bypass disconnection zones through a new routing protocol based on i) the estimation of the contact time between a vehicle and its neighbors, ii) the data load to be transferred and (iii) logs of communication errors. The objective is to ensure the availability, the reliability and the robustness of inter-vehicle communications by taking these three criteria into account in a data routing algorithm.

MOTS-CLÉS. IoV, VANETs, Communication V2V, Performances, QoS, Robustesse, Routage.

KEYWORDS. IoV, VANETs, Communication V2V, Performancese, QoS, Robustness, Routing.

\section{Introduction}

La nouvelle ère de l'Internet des Objets (Internet of Things ou IoT) a suscité l'évolution des réseaux ad hoc véhiculaires classiques (VANET) vers le paradigme de l'internet des véhicules (Internet of Vehicles ou IoV). Ce type de réseau est formé essentiellement par des unités de bord installées sur les véhicules (On Board Unit ou OBU) et les bornes installées sur les routes (Road Side Unit ou RSU). Nous appelons les types de communications possibles dans les réseaux véhiculaires "V2X", où le $\mathrm{X}$ fait référence à un véhicule $(\mathrm{V} 2 \mathrm{~V})$, une infrastructure (V2I), une personne (V2P) ou tout autre objet connecté (V2O). Grâce à ses caractéristiques, l'IoV est considéré comme une partie de l'IoT où les objets sont des véhicules qui permettent de créer une multitude de services dédiés à l'écosystème du transport intelligent [GER16, REH15]. La communication V2V est la composante principale de l'IoV, car la grande quantité de données engendrée doit être transmise d'une source à une ou plusieurs autres destinations. Pour cela les services offerts par l'IoV s'appuient beaucoup sur les véhicules en question, comme émetteur, relai et récepteur. Les véhicules s'occupent à la fois de leurs propres communications mais servent aussi de relais d'informations pour les communications entre d'autres véhicules.

La problématique consiste à étudier la disponibilité, la fiabilité et la robustesse des communications entre les agents de l'IoV. La nature dynamique de l'IoV présente des contraintes de communication à savoir des déconnexions fréquentes susceptibles d'être un obstacle pour les exécutions d'applications à bord d'un véhicule. En effet, certains véhicules se retrouvent parfois isolés sans possibilité d'émission et de réception durant une période de temps indéterminé. L’objectif de ce travail consiste à fournir aux conducteurs et aux passagers l'accès continu et exceptionnellement à jour à des données opérationnelles, fraîches, disponibles et fiables, pour répondre aux besoins sans cesse croissant en 
terme de qualité de service dans l'environnement IoV. Nous essayerons d'améliorer des solutions existantes et proposer une solution au problème permettant d'assurer la disponibilité des services, la fiabilité des communications et des données, et la robustesse de l’IoV.

La suite de cet article est structurée comme suit. Section 2 décrit un état de l'art des réseaux véhiculaires, les axes de recherche concernant l'architecture en couches de l'IoV, les obstacles qui peuvent nuire à la qualité de communication de la couche réseaux, ainsi qu'un panorama des travaux relatifs aux protocoles de routage de type multi-sauts et réactif. Section 3 explique en détail notre protocole de routage pour les communications IoV. Elle se compose de trois phases différentes : estimation de durée de contact et considération de l'équilibrage de charge, utilisation et mise à jour des logs d'anomalies de communication, calcul de routage saut par saut. Section 4 conclut l'article avec les perspectives de notre travail.

\section{Etat de l'art}

\subsection{Architecture de I’loV}

Du point de vue réseau, un système IoV est un système à trois couches [HUA13, REH13, FAN15] : application, réseaux et perception. La couche application comprend les applications de sécurité, de gestion de trafic et de confort ainsi que l'interface interactive homme-machine. La couche réseaux représente les différentes technologies de communication. La couche perception concerne tous les types de capteurs et moyens de collecte de donnée. Nous avons choisi de nous intéresser à la couche réseaux (système de communication) avec les trois axes de recherche que l'on souhaite exploiter :

- Qualité de services : déterminer les métriques de qualité de service (la fiabilité, la disponibilité et la robustesse);

- Routage : acheminer les informations dans les réseaux inter-véhiculaires sans interruption ;

- Applications, collaboration et intelligence collective : offrir une intégration complète du réseau homme/véhicule/objet/environnement via les applications IoV.

\subsection{Obstacles de communication}

Certains obstacles peuvent engendrer des déconnexions dans l'IoV comme :

- la forte mobilité des véhicules, la vitesse élevée de déplacement, et le changement rapide de la topologie du réseau ;

- la variation de la densité de véhicules dans les zones urbaines, autoroutes ou ras compagne, et l'absence de bornes RSU;

- l'utilisation des réseaux cellulaires à couverture restreinte pour les communications ;

- les attaques de type trou noir (Black Hole) que peut subir le réseau de communication ;

- les attaques par dénis de service distribué ;

- la surcharge du réseau due à la quantité d'agents communicants dans l'IoV, et la bande passante limitée ;

- l'architecture distribuée et l'accès à distance aux services offerts par l'IoV ;

- l'hétérogénéité des entités qui constituent l'IoV;

- le manque de coopération entre les agents de l'IoV et certaines infrastructures qui peuvent être des relais de communication (à cause de différents niveaux requis de sécurité par exemple); 
- la complexité et la densité de l'environnement extérieur (forêt, building, etc.);

- l'égoïsme de certains agents mobiles.

\subsection{Travaux connexes}

Il existe, pour l'acheminement des données, différents modes, stratégies et protocoles de routage, parmi lesquels nous citons le routage multi-sauts et réactif [IND14]. D'après ses caractéristiques, ce dernier s'avère adapté aux réseaux mobiles, car il assure de meilleures performances que le routage orienté source de données (en termes de rapport global de livraison de paquets et de retard de bout en bout). Par conséquent, il est adopté par la plupart des protocoles de routage ad-hoc sans fil des environnements mobiles. Figure 2.3.1 présente une taxonomie des protocoles de routage existants dans les réseaux véhiculaires. Ils sont classés selon différents modes de fonctionnement. Les algorithmes de routage dédiés aux réseaux mobiles sont souvent des protocoles multicritères et peuvent appartenir à plusieurs catégories parmi celles citées dans figure 2.3.1.

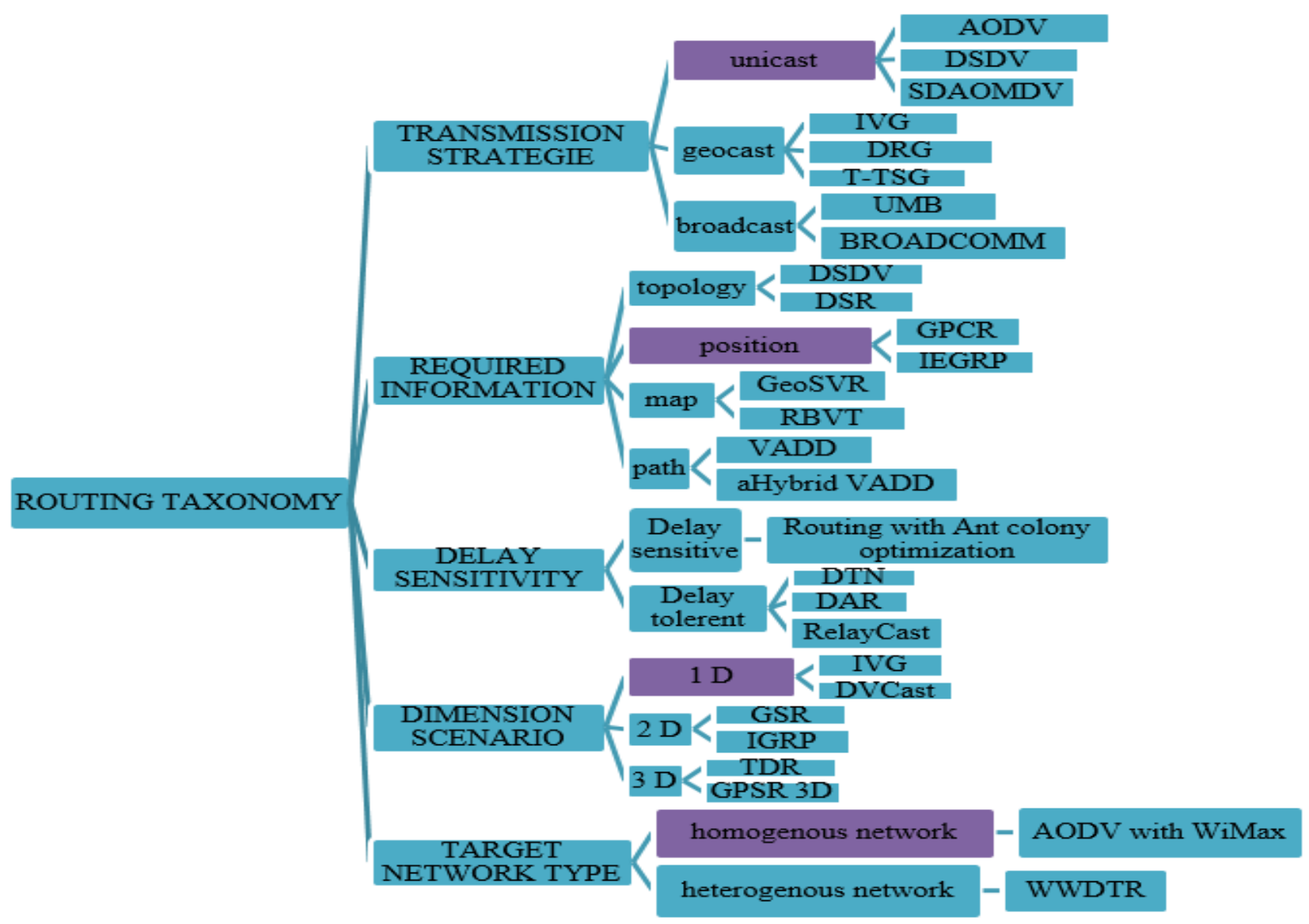

Figure 2.3.1. Taxonomie des protocoles de routage des réseaux véhiculaires

Le protocole de routage AODV (Ad hoc On Demand Distance Vector) [GHA15] prend en charge à la demande, le routage unicast et multicast, en utilisant un numéro de séquence pour chacune des routes. AODV répond à l'échec de liaison dans le réseau car il est réactif. Son principal inconvénient est que ce type de réseaux peut déterminer une route qui n'est pas forcément la route optimale. Une amélioration d'AODV, SD-AOMDV (Ad hoc On-demand Multipath Distance Vector Routing), est proposée dans [MAO12] pour traiter les caractéristiques de VANET. SD-AOMDV ajoute de nouveaux 
facteurs (vitesse et direction) au champ qui détermine le prochain saut lors du processus de découverte et de construction de routes. Le protocole AODV et sa variante sont liés à un processus de découverte de route avant le début de transmission, ce qui en fait leur principal inconvénient. Il calcule plusieurs itinéraires complets de la source jusqu'à la destination, et il devient réactif à la survenue d'un problème de communication ou à la rupture d'un lien pour la construction d'une autre route. Ce type de protocole orienté source semble inadéquat aux réseaux fortement dynamiques comme les VANETs. De plus, le calcul de plusieurs routes engendre une augmentation de délai de bout en bout du protocole.

Les auteurs de [ZHA08] proposent le protocole VADD (Vehicle-Assisted Data Delivery), qui utilise la technique "store and forwarding", où un véhicule en mouvement porte un paquet, calcule, selon un modèle de délai d'acheminement, le prochain lien qui réduit le retard de livraison puis envoie le paquet sur ce lien. À la différence des autres solutions, ce protocole s'appuie sur la mobilité prévisible des véhicules, limitée et recadrée par un modèle de trafic existant et par la structure des routes. Les auteurs proposent des variantes de VADD, qui déterminent quel lien garantit le délai de livraison de données le plus faible. Le choix du prochain lien est basé sur la localisation dans la première variante (Location VADD), et sur la direction dans l'autre variante (Direction VADD). Les auteurs ont également combiné les deux critères de sélection dans Hybrid VADD. Leurs résultats expérimentaux montrent parmi les protocoles VADD proposés, le Hybird VADD présente de meilleures performances que Location VADD, et Direction VADD [CHEN15]. Ils prouvent également que les protocoles VADD proposés surpassent les protocoles Epidemic, GPSR (Greedy Perimeter Stateless Routing) with buffer et Dynamic Source Routing (DSR), en termes de ratio de livraison de paquets, de délai de livraison et de surcharge de protocole.

Un autre protocole qui se base sur les informations des structures routières est le protocole RBVT-R (Road Based Vehicular Traffic Reactive Protocol) [NZO09], un protocole réactif qui exploite le trafic routier en temps réel pour créer des itinéraires grâce aux segments routiers dit "connectés", i.e. forte probabilité de connectivité réseau. Il effectue alors une élection distribuée du prochain saut en se basant sur une fonction de priorisation multicritères dont la densité de véhicules voisins [KARP00].

Le protocole de routage GPSR [CHEN15, KARP00, SHR12] (Greedy Perimeter Stateless Routing for wireless networks) est basé sur la position géographique ce qui permet de l'adapté aux VANETs. Un nœud choisit le prochain relai en fonction des informations de position qu'il reçoit périodiquement de ces voisins, et de la destination. Sa particularité consiste à récupérer les informations des véhicules voisins par un équipement de positionnement GPS, plutôt que d'obtenir un grand nombre d'informations de routage, et de maintenir une table de voisinage ainsi qu'une table de routage. La stratégie de transmission combine une stratégie d'acheminent avide avec une stratégie d'acheminement périmétrique. Une amélioration de GPSR, le protocole I-GPSR (Improved GPSR) a été proposé dans [KAU16], qui ajoute comme critère de décision à la vitesse de déplacement et l'orientation des véhicules. Le protocole I-EGRP [KARP00] est adapté aux environnements à faible débit en changeant dynamiquement ses décisions de routage à l'aide de chaque borne RSU détectée. L'inconvénient de ce protocole est que son choix est déterminé en fonction de la présence d'une infrastructure fixe.

Comme autre protocole de routage multicritères, le MUDDS (Multi-metric Unicast Data Dissemination Scheme) proposé dans [CHA12] est un schéma de propagation de données unicast. Il détermine son choix du prochain relai grâce à deux métriques calculées localement à savoir : le taux de réception de paquets (PRR), et la disponibilité des liens (LA). Il fonctionne en deux phases. Lors de sa première phase, il adapte sa portée de transmission pour garantir un PRR maximum en fonction de 
l'état du réseau en termes de congestion et de densité de communication. Puis effectue en second lieu la transmission de messages sur la base de la métrique LA. L'utilisation du PRR garantit la fiabilité du transfert des messages et le choix du relai basé sur LA réduit le nombre de sauts, et évite la fragmentation du réseau.

Le protocole MA-DSDV [HAR14] (Multi Agent Destination-Sequenced Distance Vector routing) est une amélioration du protocole de routage à vecteur de distance, modélisé en système multi-agents. Il fournit l'itinéraire avec le plus petit nombre d'agents relais. La partie multi-agents permet en cas de défaillances, d'agir de manière autonome et de communiquer entre les agents les informations nécessaires à la reprise du routage à tous ses voisins.

Le protocole GVGrid [SUN06, NZO09] est orienté qualité de service QoS, construit un chemin à la demande, d'une source vers des véhicules positionnés dans une région géographique déterminée. Il divise la carte géographique en plusieurs grilles sur laquelle il met en évidence les liens entre les nœuds. Le choix des nœuds relai se fait selon le nombre de déconnexions du lien qui le relie à l'émetteur. L'objectif de GVGrid est de maintenir une route robuste qui résiste à la mobilité des véhicules. Les résultats expérimentaux ont montré que GVGrid pourrait fournir des itinéraires avec une plus longue durée de vie, par rapport à d'autres protocoles de routage pour VANETs. Son principal inconvénient est qu'il n'offre aucune garantie de délais optimaux qui est une métrique de qualité de service requise dans les VANETs.

La caractéristique supplémentaire qu'apporte l'IoV par rapport aux réseaux VANETs traditionnels est la forte probabilité d'avoir un réseau et des objets connectés complètement hétérogènes. En plus des problèmes classiques du routage homogène, l'utilisation des réseaux hétérogènes engendre un temps supplémentaire "Hand-over" qui représente le temps de passage d'une technologie d'accès réseaux à une autre. Les auteurs de [SHA11] proposent une stratégie répartie de transfert vertical d'une technologie de communication à une autre appelée "Optimal Vertical Handoff" [SHA11].

Dans un réseau hétérogène et grande échelle, la notion de clustering est très convoitée par la communauté. Lorsque les nœuds sont regroupés et que chaque cluster choisi son cluster Head, la question restante pour un nœud source est de choisir quel véhicule jouera un rôle de proxy, c'est-à-dire une passerelle vers l'infrastructure d'une autre technologie de communication [KARP00]. Une stratégie de sélection proposée dans [LAB12] offre une priorisation des technologies disponibles. Les auteurs optent par exemple pour la technologie LTE-Advanced (Long Term Evolution-Advanced, une norme de réseau de téléphonie mobile de $4{ }^{\text {ème }}$ génération) comme deuxième interface sans fil, qui attache directement la source à l'infrastructure. En vue d'améliorer le coût de communication, les auteurs conçoivent également un algorithme distribué, adaptatif et multicritères pour la sélection de passerelle d'un réseau à un autre, appelée "QoS based Gateway Selection". Cet algorithme s'avère meilleur que l'approche déterministe utilisant le cluster Head comme passerelle.

Enfin des auteurs de [LI12] proposent une stratégie qui consiste à sélectionner une partie des véhicules en tant que passerelles mobiles pour relier les différents composants du réseau hétérogène. Des coalitions de nœuds sont créées en se basant sur la théorie des jeux qui stimulent les nœuds à choisir la meilleure coalition pour passer d'une passerelle à une autre, et à rejoindre les coalitions qui peuvent maximiser efficacement le débit de données.

Les travaux analysés montrent que pour satisfaire les contraintes de communication dans les réseaux fortement dynamiques, une stratégie de routage doit prendre en compte de multiples critères, quelle 
que soit la catégorie à laquelle elle appartient. Nous remarquons que chaque protocole tente d'équilibrer un certain nombre de paramètres pour optimiser principalement trois métriques, à savoir la fiabilité du routage, le délai d'acheminement de bout en bout, et le ratio de livraison de paquets.

\section{Protocole de routage multicritères}

Nous avons besoin de protocoles de routage bien défini pour résoudre l'ensemble des problèmes causés par la nature grande échelle et l'hétérogénéité de l'IoV. Il serait optimal de commuter entre les technologies de communications lorsqu'un problème survient, car cette approche offre en théorie de meilleures performances. Néanmoins elle présente des défis de stabilité et beaucoup d'autres contraintes (matérielles, juridiques, etc.).

On s'intéresse à la partie algorithmique où il s'agit principalement d'apporter des méthodes efficaces, fiables, robustes et optimisées pour la transmission sécurisée de paquets de données en fonction de l'évolution de la topologie et de la mobilité du réseau. Il s'agit de garantir la connexité du réseau en dépit de la mobilité. Le mouvement des véhicules du réseau, même minime, peut ainsi provoquer la partition physique du réseau en plusieurs sous-réseaux. Le déplacement d'un véhicule sur un chemin de routage modifie les possibilités de routage dont dispose ce véhicule. Les voisins précédemment joignables peuvent devenir hors de portée de communication alors que d'autres précédemment inconnus deviennent joignables. Le déplacement des véhicules entraine donc à la fois la suppression et la création de chemins de routage possibles, il s'agit d'en tenir compte lors des choix de routage. La connaissance de la durée de contact inter-véhicules est alors un paramètre important et utile pour les véhicules [ALS16], car il permet de contourner et d'anticiper de manière proactive une rupture de liaison prématurée. De plus, il représente un des paramètres d'entrée dans le processus d'acheminement, car il permet la sélection du prochain saut optimal, et la construction locale et proactive d'un chemin robuste pour la transmission des données. Néanmoins, en raison de la grande mobilité des véhicules, et de la complexité des modèles de mobilité, l'estimation de la durée des liaisons dans les réseaux mobiles, et dans les réseaux véhiculaires reste un sujet de recherche ouvert.

C'est en se basant sur ces constatations que nous avons proposé un algorithme de routage qui va d'abord tirer parti de l'estimation des durées de contact pour construire les chemins de routage des paquets. Il anticipe au maximum les choix de routage et d'agents relais sur chaque saut de manière locale. En plus de l'estimation des durées de contact, nous ajoutons une deuxième entrée au processus de sélection du prochain relai qui est le paramètre d'équilibrage de charge. Le véhicule transmet luimême dans une variable, sa charge en nombre de paquets à relayer. Enfin, avant de choisir un relai, nous vérifions que ce dernier n'a pas de problèmes de connectivité.

\subsection{Applications et services}

Les réseaux véhiculaires consomment un gros volume de données qu'ils enrichissent dans différents types d'applications comme :

1. La sécurité routière : informations sur les conditions météorologiques, sur des obstacles sur la route, prévention d'un véhicule dangereux, localisation d'un véhicule volé ;

2. La gestion de trafic routier : informations sur les congestions, choix d'itinéraire, régulation de la vitesse en fonction des feux de circulation, et les feux tricolores en présence des piétons ;

3. L'accès à des contenus multimédias via les hots spots Internet en passant d'une voiture à une autre jusqu'au point d'accès le plus proche : vidéo streaming, fichier mp3, jeux en ligne ; 
4. Le suivi de flottes de marchandises : synchronisation de convois, bus, taxi, etc.

Bien que ces applications aient des objectifs distincts, elles ont néanmoins les mêmes contraintes au niveau des réseaux de communications. Elles doivent pouvoir être alimentées continuellement par ses utilisateurs, par les entités du réseau véhiculaire ou par les RSU. Nous avons deux types de communication bien distincts :

Communication de type "Forward" : lorsqu'un véhicule se déplace, il acquiert un historique des évènements sur le tronçon traversé, qu'il souhaite partager avec les véhicules entrant dans ce tronçon afin d'en tirer profit. Dans ce cas, l'information est diffusée aux véhicules se trouvant dans le tronçon déjà traversé (information dans le sens inverse de déplacement du véhicule) pour être utile aux utilisateurs. Ce type de communication est orienté communications évènementielles. Figure 3.1.1 illustre ce type de communication.

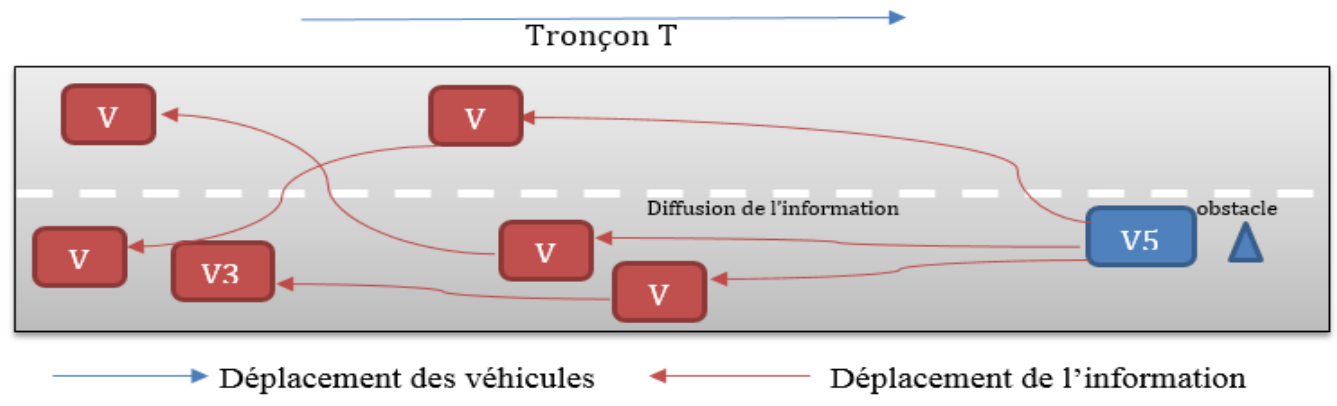

Figure 3.1.1. Exemple de communication Forward

Communication de type "Request/Reply" : nous nous intéressons aux communications où un véhicule émet une requête d'accès à un service $\mathrm{Web}$ par exemple, puis rentre dans une zone noire. La réponse à sa requête sera alors transférée en $\mathrm{V} 2 \mathrm{~V}$. Le premier véhicule qui commence le transfert de la réponse dispose d'une connexion Internet. Dans un premier temps, nous supposons connaitre l'adresse IP du véhicule à qui on doit transmettre l'information (destination), et du premier véhicule relai qui dispose d'une connexion Internet. Ce cas est illustré dans figure 3.1.2.

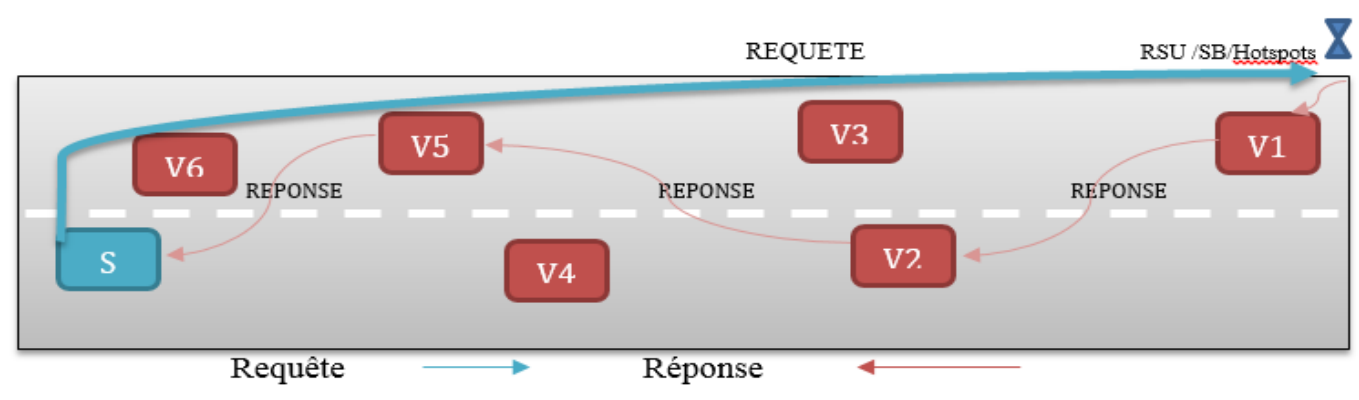

Figure 3.1.2. Exemple de communication Request/Reply

Notre travail vise à garantir l'acheminement de données vers un véhicule spécifique, voir, une infrastructure RSU, et assurer une communication entre une source et une destination, pouvant améliorer les performances des types d'applications citées ci-dessous. Notre algorithme est générique et peut s'appliquer aux différentes applications comme par exemple : 
- Faire parvenir un diagnostic d'une panne à un garage de proximité dont on connait l'emplacement géographique via $\mathrm{V} 2 \mathrm{~V}$;

- Alimenter une station météorologique fixe en temps réel via $\mathrm{V} 2 \mathrm{~V}$;

- Télécharger du contenu multimédia d'un point d'accès fixe via V2V ;

- Profiter d'une connexion Internet haut débit et stable pendant un voyage, via V2V ;

- Relayer un message d'urgence émanant d'un véhicule, en l'absence de couverture cellulaire ;

- Assurer le suivi des véhicules de location, ou de flotte de marchandise ;

Il s'agit dans notre travail, d'une solution pour les communications "1 to 1" d'une source mobile à une destination fixe ou mobile, via des véhicules intermédiaires dans le cadre d'application IoV, sur un plan à une dimension.

\subsection{Protocole de routage multicritères dans les communications loV}

Le but de notre démarche est de minimiser les ruptures de liens pendant les communications, par conséquent réduire les coûts de résolution et la redondance de certains messages systèmes. Lorsque des véhicules entrent en contact, par exemple, pour alimenter une application de gestion de trafic, prévenir d'un obstacle, transmettre des informations en temps réel à une station météorologique, ou même de transmettre une donnée récupérée sur le réseau internet (P2P), les liens de communication seront maintenus pour de faibles durées. Ces communications opportunistes limitent le volume échangé de données sur une liaison et réduit la fiabilité de transmission. Pour pouvoir optimiser l'acheminement des paquets de données, nous devons estimer, au préalable, les durées de contact entre les entités de l'IoV, et en tenir compte dans le processus de dissémination. On considère un tronçon $\mathrm{T}$ délimité par deux intersections, où une intersection est définie par une signalisation du code de la route, une entrée d'autoroute, ou tout autre obstacle engendrant un changement de régime de déplacement. Figure 3.2.1 illustre un exemple de scénario d'échange de données sur une autoroute, où l'on observe comment la notion de voisins est redéfinie et comment sélectionner un relais parmi d'autres.

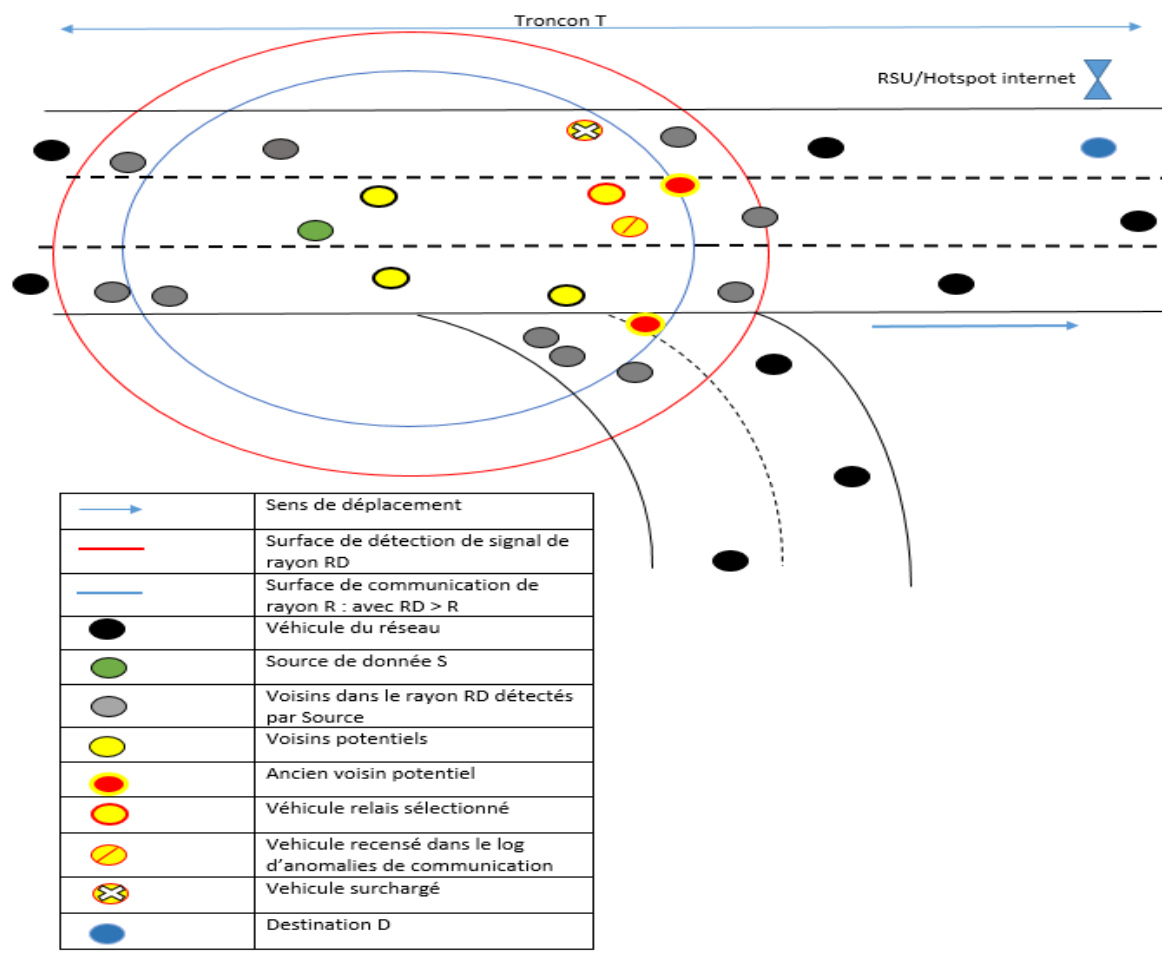

Figure 3.2.1. Exemple d'exécution de notre stratégie de choix du meilleur voisin 
Soit $\mathrm{V}$ l'ensemble des véhicules du réseau. $\mathrm{V}_{1}$ est un véhicule qui émet un besoin de communiquer avec un autre véhicule pour lui transmettre des données, ou alors pour enrichir, avec de nouvelles informations, une application distribuée ou stockée sur un serveur distant. Pour cela le véhicule s'appuie sur des communications $\mathrm{V} 2 \mathrm{~V}$. Tant que $\mathrm{V}_{1}$ a besoin de communiquer, il exécute notre algorithme de routage géographique à un intervalle de temps $t_{m e}$ (donnée par la formule [4]). Le paramètre $t_{m e}$ est lié à la dernière durée de contact estimée, influencé par le taux de fiabilité de communication du relai choisi précédemment.

Chaque véhicule sélectionné comme relai, exécutera à son tour l'algorithme de routage pour choisir son prochain relai. Le processus de routage et l'exécution de l'algorithme de routage se termine lorsque les paquets atteignent la destination finale. Notre algorithme général se décompose en trois phases.

\subsubsection{Phase 1 : Estimer la durée de contact}

Cette étape a pour but d'estimer des durées de contact entre les véhicules dans un voisinage.

\section{Hypothèses}

Chaque véhicule connait ses coordonnées géographiques (GPS), et dispose d'interface de communication sans fil. Un message de type Beacon: $\left.\mathrm{V}_{2}<x_{2}, y_{2}, v_{2}, \overrightarrow{d i}, C_{2}, i d_{2}\right\rangle$ et de taille réduite, est envoyé au préalable et à intervalle régulier, par un véhicule à ses voisins dans un rayon de détection de signal $R D$, pour transmettre ses informations locales. Ce message de taille relativement petite peut être associé à un autre type de message de contrôle pour réduire le coût des messages échangés. Les variables $x, y$ représentent les coordonnées géographiques de l'agent mobile, $v$ est la vitesse de déplacement, le vecteur $\overrightarrow{d i r}$ donne le sens de déplacement, $c$ représente la charge libre du véhicule en nombre de paquets, $i d$ est l'identifiant de l'agent en l'occurrence son adresse IP. Ces messages ainsi échangés permettent d'avoir une vue quasi globale du réseau pour chaque véhicule.

\section{Description}

On élabore, grâce aux informations des messages Beacon, un algorithme distribué, qui estime des fenêtres de temps de connexion, durant lesquelles une communication est, ou sera, fiable et disponible pour un couple de véhicules.

Soient $\mathrm{V}_{1}<x_{1}, y_{1}, v_{1}, \overrightarrow{d i r}, C_{1}, i d 1>$ et $\mathrm{V}_{2}<x_{2}, y_{2}, v_{2}, \overrightarrow{d i r}, C_{2}, i d_{2}>$ deux entités du réseau véhiculaire roulant sur un tronçon $\mathrm{T}$. Pour estimer la durée de contact entre $\mathrm{V}_{1}$ et $\mathrm{V}_{2}$, la distance entre les deux entités doit être inférieure ou égale au rayon de transmission $\mathrm{R}$ du matériel radio à bord des véhicules. Cette condition est donnée par l'inégalité ci-dessous :

$$
\sqrt{\left(x_{2}-x_{1}\right)^{2}+\left(y_{2}-y_{1}\right)^{2}} \leq \mathrm{R} \quad[1]
$$

$\mathrm{R}$ est le rayon d'un cercle, qui représente la surface maximale de communication couverte par la technologie de communication radio utilisée par un véhicule.

Etant donné que la vitesse des véhicules n'est en pratique jamais constante, on utilisera non pas la vitesse de déplacement du véhicule par rapport à la route mais la vitesse relative de chaque véhicule par rapport à un autre véhicule. Dans le cas où les deux véhicules ont la même vitesse $v_{1}=v_{2}$, leur 
durée de contact (DDC) est égale au rapport de la distance sur la vitesse de déplacement d'un des véhicules. Dans le cas contraire, le temps de la durée de contact est donné par l'expression ci-dessous :

$$
\frac{\sqrt{\left(x_{2}-x_{1}\right)^{2}+\left(y_{2}-y_{1}\right)^{2}}}{\left|v_{2}-v_{1}\right|} \text { avec } v_{2} \neq v_{1}
$$

Ce qui implique que la durée de contact DDC entre un couple de véhicule $\mathrm{V}_{1}$ et $\mathrm{V}_{2}$ appartient à l'intervalle : $\left[0, \frac{\mathrm{R}}{\left|v_{2}-v_{1}\right|}\right]$

Après avoir calculé cette durée de contact, un véhicule $V_{1}$ aura comme résultat un ensemble de voisins potentiels avec les informations $\left\langle I d, v_{i}, \overrightarrow{d i r}, C_{i}, D D C_{i}\right\rangle$ correspondant à chacun. Nous redéfinissons la notion de voisinage dans cet algorithme. Un véhicule $V_{i}$ est considéré comme voisin d'un véhicule $\mathrm{V}_{1}$ si et seulement si :

- Il détecte son signal ;

- Il se déplace dans le même sens, et dans la même direction que l'autre véhicule ;

- Il a une distance euclidienne $\mathrm{d}\left(\mathrm{V}_{\mathrm{i}}, \mathrm{V}_{1}\right)$ inférieure au rayon $\mathrm{R}$.

Après l'estimation des durées de contact d'un véhicule avec ses voisins, il communiquera cette liste ordonnée de voisins potentiels en fonction des besoins de notre stratégie.

Dans notre travail nous prendrons, sur le tronçon $\mathrm{T}$, un point d'origine fixe $\mathrm{O}$, auquel seront référencées les coordonnées $\left(x_{\mathrm{i}}, y_{\mathrm{i}}\right)$, et qui servira à déterminer le sens de déplacement des véhicules. Quant à la direction de déplacement $d i r_{i}$, dont on a besoin pour la sélection des relais, nous prendrons les véhicules $\mathrm{V}_{\mathrm{i}}$ voisins qui répondent à l'équation suivante :

$$
\overrightarrow{\operatorname{dir}}_{v i}=k \overrightarrow{\operatorname{dir}}_{v 1} \text { avec } k>0
$$

Dans notre proposition, nous avons décidé de ne pas fixer la technologie de communication. L'importance de la technologie de communication pour notre contribution réside dans les paramètres qu'il offre en l'occurrence la portée du de la transmission (rayon R), ainsi que la longueur du segment de données qu'il porte. Nous considérons des paquets unitaires de données de taille fixe, qui nécessitent, en fonction du débit offert par la technologie de communication, un temps de transmission direct $T p$ d'un véhicule $\mathrm{V}_{1}$ à un véhicule $\mathrm{V}_{2}$.

L'Algorithme d'Estimation des Durées de Contact (AEDC) est exécuté pour chaque véhicule afin de redéfinir son voisinage selon nos besoins de communication, et estimer les durées de contact avec chaque voisin. Il renvoie alors une collection de voisins "Neighbors" avec des informations de type "Beacon_DDC". Le pseudo code de la fonction AEDC est illustré dans figure 3.2.1.1. L'objet de type "Beacon_DDC " regroupe les informations relatives à chaque voisin éligible tels que l'identificateur Id du véhicule qui émet cet objet, sa direction de déplacement, sa vitesse de déplacement, sa charge disponible, et sa durée de contact. 


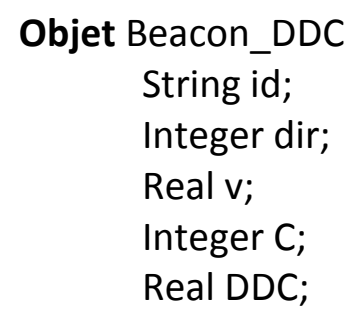

\section{End Objet}

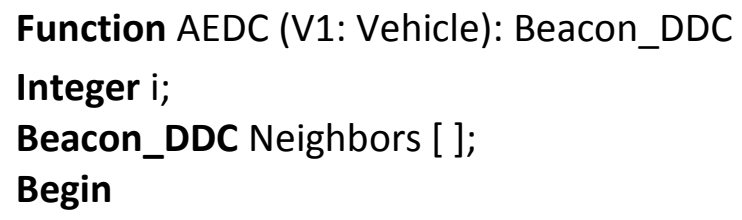

End for;

Return (Neighbors) ; // Beacon_DDC is the type of Neighbors []

End.

Figure 3.2.1.1. Algorithme d'Estimation des Durées de Contact entre V1 et son voisinage

\subsubsection{Phase 2 : Mettre à jour des logs d'anomalies de communication}

Nous utilisons sur un tronçon $\mathrm{T}$, des logs distribués sur chaque véhicule, qui seront accessibles en écriture et en lecture par le véhicule qui le sauvegarde, et uniquement en écriture par les autres véhicules.

\section{Exploitation des logs}

Lorsqu'un véhicule rencontre une anomalie de communication avec un autre véhicule, subit une déconnexion par exemple, son fichier log est mis à jour, avec des informations relatives à l'anomalie détectée. Les informations primaires sont le/les véhicules concernés, l'instant et/ou la durée de la déconnexion, la zone non couverte, la densité de véhicules dans cette zone, et éventuellement la cause de la déconnexion. Cette phase va détecter les zones noires, et permettre aux véhicules d'agir en conséquence lors du choix des véhicules intermédiaires par lesquels transiteront les données. Les zones 
noires sont recensées dans le log de chaque véhicule. Elles sont mises à jour soit par le véhicule luimême s'il est en mesure de le faire, ou par les voisins directs par distribution partielle de leurs logs.

La détection d'anomalies de communication de $\mathrm{V}_{1}$, par les véhicules voisins Vi se fait soit par :

- La rupture prématurée d'une communication ;

- La non-réception d'acquittement des messages envoyés ;

- La variation aléatoire des messages Beacon d'un véhicule sensé être voisin.

Nous présentons le pseudo code de la procédure de mise à jour des logs d'anomalies de communication, qui est exécutée par chaque véhicule. Cette procédure est donnée dans figure 3.2.2.1.

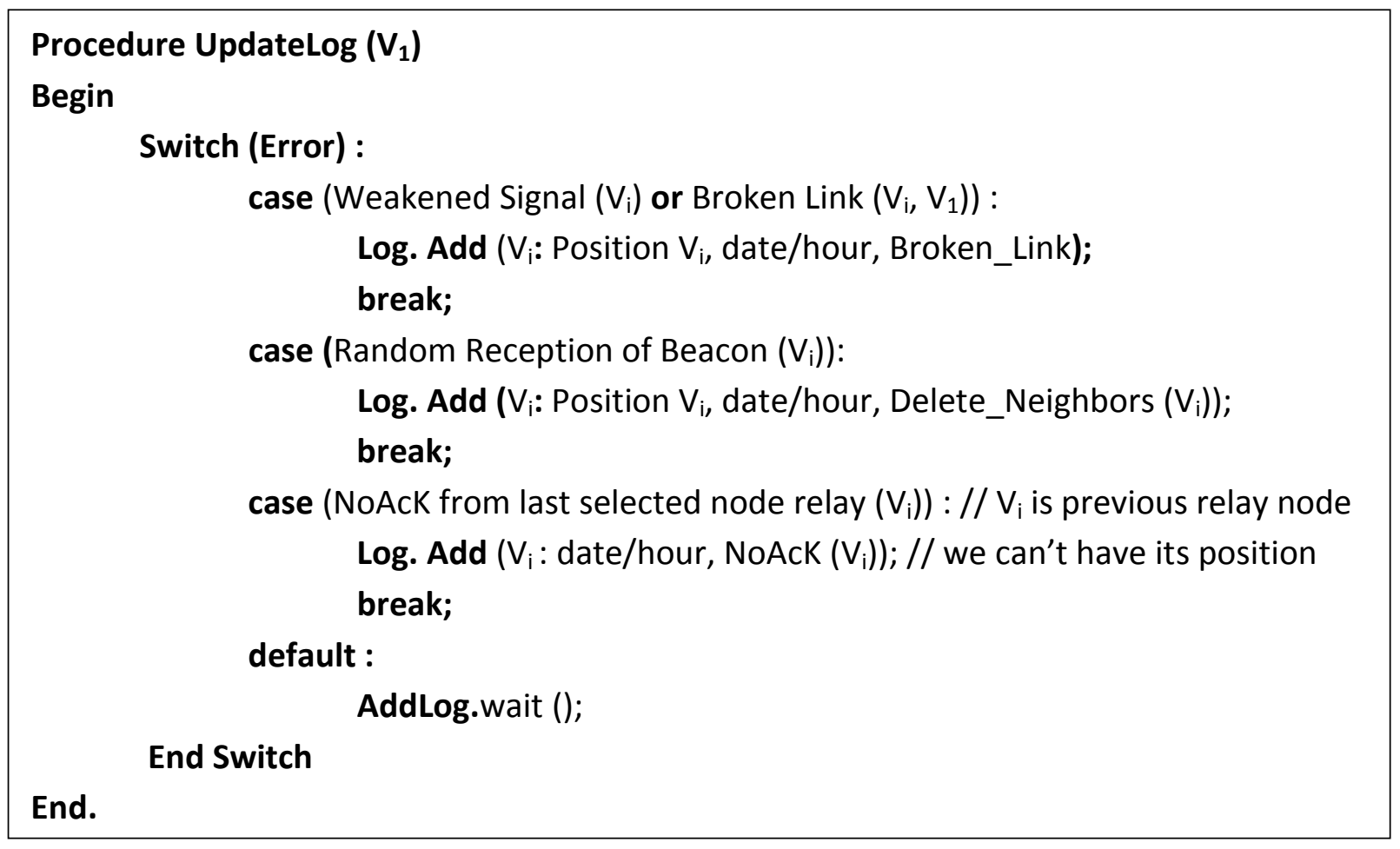

Figure 3.2.2.1. Stratégie de mis à jour des logs d'anomalies de communication

\subsubsection{Phase 3 : Déterminer le routage géographique}

A un niveau plus bas du standard de communication, la couche réseau nous permettra de faire une combinaison entre un routage IP et un routage géographique. Notre protocole est réactif et adaptatif, i.e. le chemin de transition des paquets d'une source à une destination est construit au fur et à mesure que le message se déplace. Les principales métriques qui nous permettent de déterminer une stratégie de routage sont la rupture prématurée d'une communication, la distance, la densité de communication, l'appartenance à un cluster, le débit et l'état des liens. Notre stratégie amène deux autres métriques de décision qui sont la durée de contact et le taux de référencement dans le log d'anomalies de communications.

\section{Hypothèses}

- Chaque véhicule est coopératif et dispose d'une interface de communication ;

- La table de localisation des voisins [ATE10] des véhicules est enrichie grâce aux messages Beacon;

- On connait au préalable la direction de la destination finale, ainsi que son identifiant. 


\section{Description}

Pour avoir un système complet, on intègrera via une interface communicante, les résultats d'estimation des durées de contact à notre algorithme de routage, car bien qu'ils soient des modules séparés, ils restent complémentaires. Pour aboutir aux objectifs fixés au départ, les résultats de la phase 1 et phase 2 contribueront à la décision du prochain relais d'acheminement des paquets. Pour mieux contrer les conséquences de déconnexions fréquentes, on choisit d'orienter notre protocole vers une approche locale et progressive. Le chemin de relayage de données est construit au fur et à mesure que l'on avance, en respectant les contraintes temporelles et réelles du véhicule.

Lorsqu'un véhicule est émetteur ou, reçoit des paquets à retransmettre, il exploite les résultats des phases précédentes pour choisir parmi son voisinage le relai le moins chargé avec une durée de contact optimale et ne figurant pas dans le log des anomalies de communication. Cette stratégie est applicable également lorsqu'une rupture de lien survient, ou lorsqu'un véhicule est notifié par une anomalie enregistrée dans un fichier log et qui concerne un véhicule avec qui il communique actuellement.

Chaque fichier log contient une structure de donnée qui enregistre les véhicules responsables d'anomalies de communication. Lors de la construction de lien de communication les véhicules :

- récupèrent la liste des voisins potentiels ;

- comparent les variables $C_{i}$ d'équilibrage de charge ;

- consultent le log d'anomalies de communication.

Grâce à $C_{i}$, nous choisirons les relais de manière à équilibrer la charge entre les voisins, et minimiser la perte des paquets due à la surcharge de certains relais. Grâce à la $D D C$ du relai choisi, et au temps d'émission d'un paquet $T p$, influencé par d'autres évènements, éventuellement les notifications du log, nous déterminons en fonction du paramètre $\alpha$, le temps moyen $t_{m e}$ de la prochaine réexécution de notre algorithme d'estimation de durée de contact qui est donné par l'équation suivante :

$$
t_{m e}=t_{0}+D D C_{i}-\alpha \text { avec } \alpha \in R
$$

Le paramètre $\alpha$ détermine la durée écoulée avant qu'une anomalie ne survienne.

L'algorithme principal RGDCL (Routage Géographique avec Durée de contact Charge libre et Log) (figure 3.2.3.2) fait appel à la fonction Max_Min (figure 3.2.3.1) qui nous permet d'ordonner les voisins éligibles de manière décroissante, en fonction du nombre de paquets NB qu'on peut envoyer à chacun des voisins. Ce nombre de paquets à envoyer doit respecter la charge libre du véhicule et s'approcher du nombre de paquets à envoyer durant la DDC calculée.

Pour chaque véhicule la fonction Max_Min est appelée avec comme paramètre la collection "Neighbors". Elle retourne une collection "Max_Min_Neighbors " de type "Eligible_Neighbor" triée selon l'ordre décroissant de leur capacité de charge à recevoir.

Chaque objet "Eligible_Neighbor" regroupe l'identifiant (id) et la valeur calculée "min" de chaque voisin éligible 


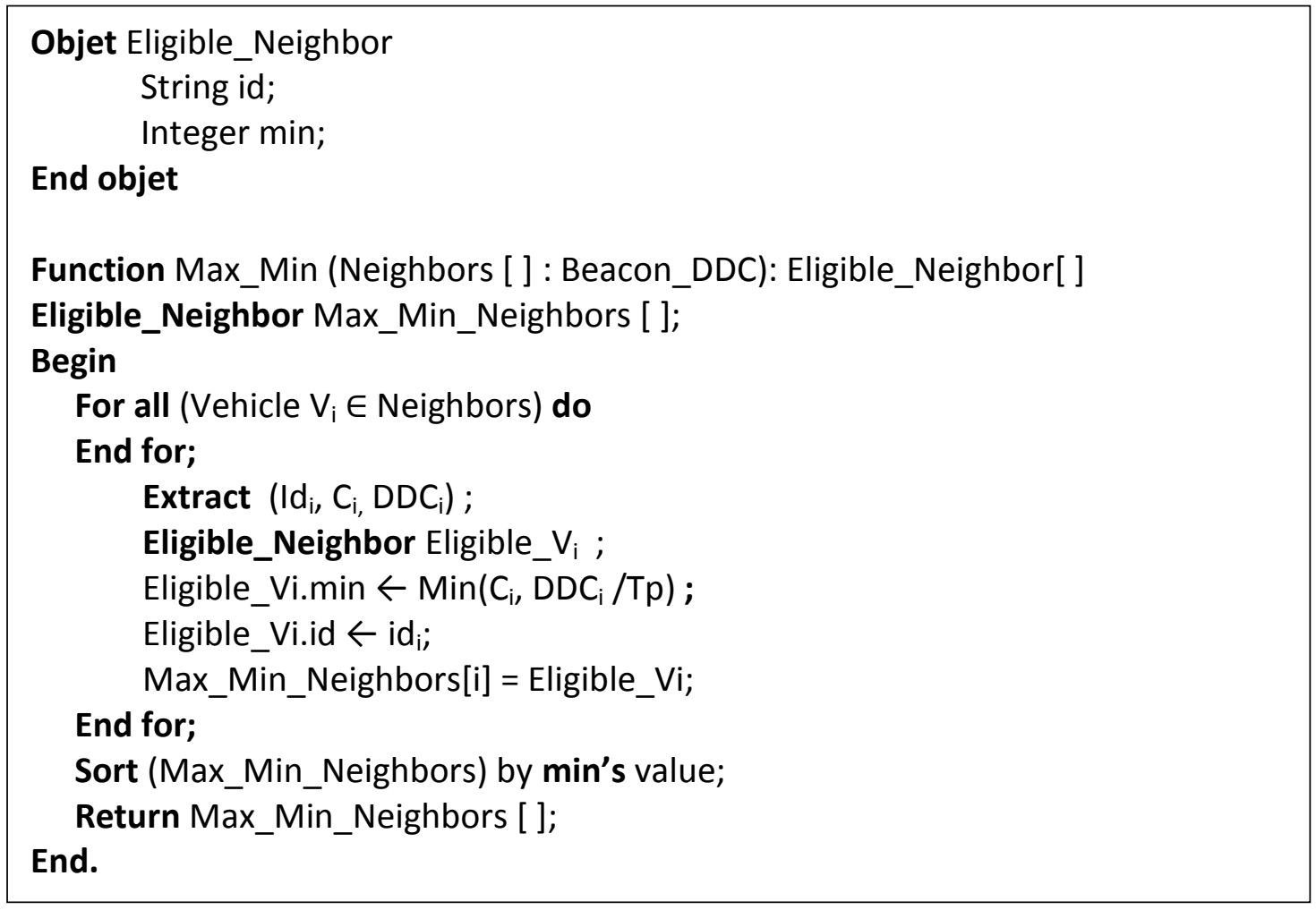

Figure 3.2.3.1. Fonction Max_Min qui retourne une collection ordonnée des véhicules éligibles

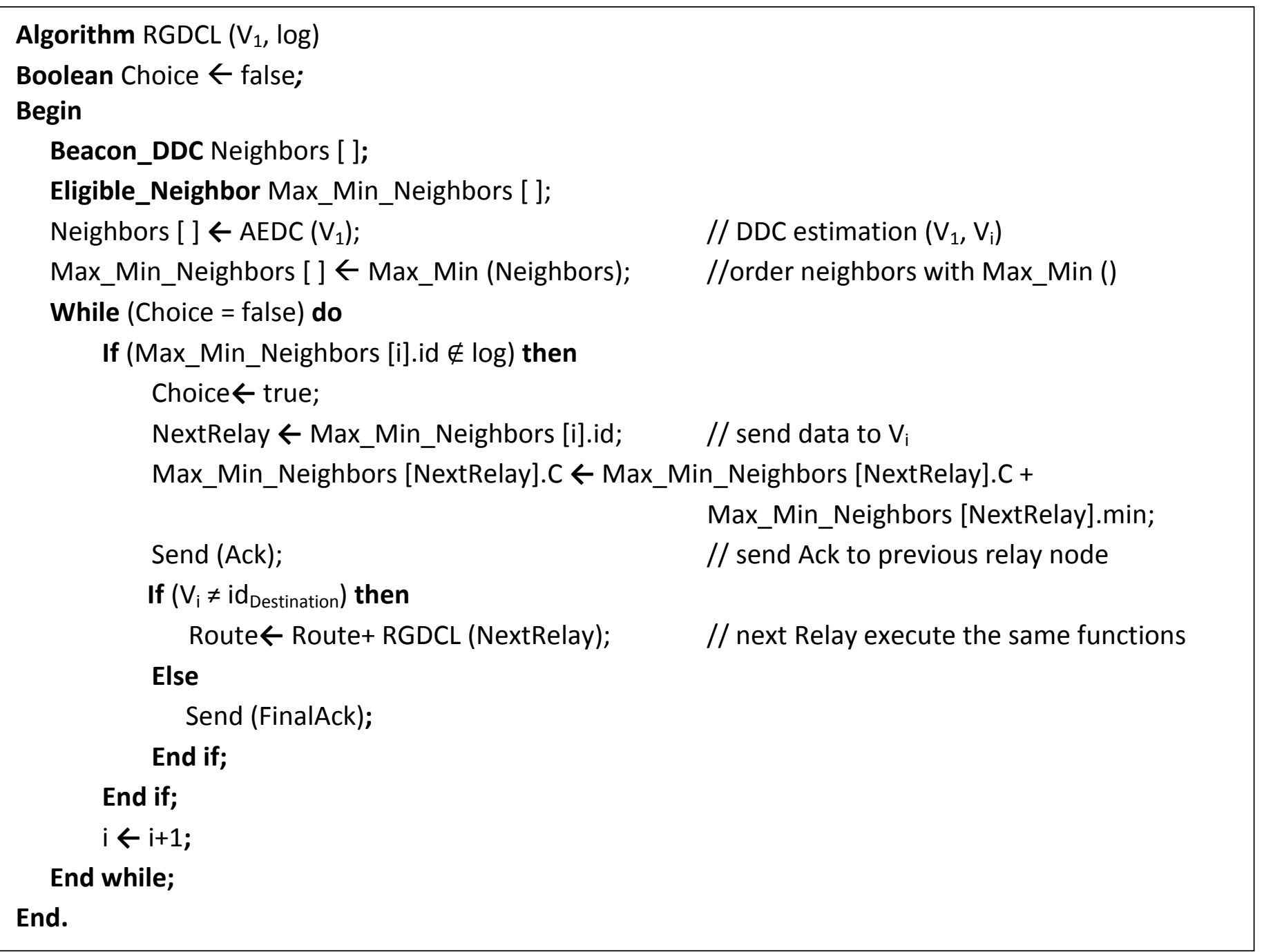

Figure 3.2.3.2. Algorithme $R G D C L$ de routage géographique multicritères 
L'algorithme RGDCL fait appel à la fonction AEDC et à la fonction Max_Min, et sélectionne enfin le prochain relai en fonction du Log. Il acquitte ensuite son émetteur pour les paquets qu'il vient de relayer, et attend l'acquittement du prochain relai sélectionné. La notion d'acquittement mentionnée dans RGDCL permet de mettre à jour le log d'anomalies de communication.

Tableau 3.2.3.3 fournit un exemple des valeurs des $V_{i}, i \in[1,8]$, les nouveaux voisins du véhicule $V_{0}$, définis selon notre stratégie. Pour chaque $\mathrm{V}_{\mathrm{i}}$, on calcule le nombre de paquets que $\mathrm{V}_{0}$ peut lui transmettre en fonction de :

- La durée de contact : $N B d=D D C\left(V_{0}, V_{i}\right) / T_{p}$

- La charge libre $C_{i}$ qui nous donne le nombre de paquets que $V_{i}$ est capable de recevoir de $V_{0}$.

Pour éviter la rupture de liens pendant les communications, la perte des paquets et la surcharge de certains relais, la fonction Max-Min détermine le nombre de paquets à envoyer sur chaque lien $\left(\mathrm{V}_{0}\right.$, $\mathrm{V}_{\mathrm{i}}$ ). Sur l'ensemble de ces liens nous choisirons le lien qui permet l'envoi du plus grand nombre de paquets NB. Ce choix est exprimé par la formule ci-dessous :

$$
\operatorname{Max}\left\{N B i / N B \in \operatorname{Min}\left(C i, \frac{D D C i}{T p}\right)\right\} \quad \text { avec } i \in[1, n] \quad[6]
$$

Donc l'algorithme va classer les voisins en ordre $\left(\mathrm{V}_{7}, \mathrm{~V}_{6}, \mathrm{~V}_{1}, \mathrm{~V}_{4}, \mathrm{~V}_{5}, \mathrm{~V}_{2}, \mathrm{~V}_{3}, \mathrm{~V}_{8}\right)$ et leur présence dans le fichier log des anomalies sera vérifiée afin de choisir le prochain élément éligible :

\begin{tabular}{|c|c|c|c|c|c|c|c|c|}
\hline Voisin de $\mathrm{V}_{0}$ & $\mathrm{~V}_{1}$ & $\mathrm{~V}_{2}$ & $\mathrm{~V}_{3}$ & $\mathrm{~V}_{4}$ & $\mathrm{~V}_{5}$ & $\mathrm{~V}_{6}$ & $\mathrm{~V}_{7}$ & $\mathrm{~V}_{8}$ \\
\hline NB (paquets) & 15 & 8 & 25 & 9 & 11 & 14 & 21 & 12 \\
\hline C (paquets) & 10 & 9 & 5 & 16 & 8 & 13 & 22 & 0 \\
\hline NB = Min (NBd, $C)$ & 10 & 8 & 5 & 9 & 8 & 13 & 21 & 0 \\
\hline
\end{tabular}

Tableau 3.2.3.3. Exemple de voisinage

\section{Conclusion et perspectives}

Afin d'acheminer les données volumineuses engendrées par l'IoV, nous avons besoin de processus de dissémination de données fiable et une connectivité irréprochable. Notre objectif est d'assurer la fiabilité et la robustesse des communications dans les réseaux véhiculaires car ces dernières sont intermittentes à cause de la mobilité et de la vitesse des véhicules ou de la présence d'obstacles. L'acheminement des informations dépend entièrement de la fiabilité de ces communications.

Afin de répondre aux exigences du système et de contourner les contraintes de communication, une solution consiste à mettre en œuvre une stratégie de communication distribuée, réactive et adaptative. Cette stratégie de communication s'appuie sur les véhicules eux même. Pour communiquer entre eux, les véhicules doivent définir un routage permettant de faire face aux fragmentations fréquentes caractérisant ce type de réseaux. 
Dans notre proposition, nous commençons par quantifier les durées de contact entre les véhicules. Pour cela, nous proposons un modèle analytique pour la durée de contact entre un véhicule et chaque voisin, sur le rayon de transmission $\mathrm{R}$, en se basant sur une distribution uniforme du flux de véhicules entrant dans une zone d'autoroute, à une période de la journée.

Nous implémenterons en suite ce modèle, sur un flux de véhicules, avec un SMA (Système Multi Agents) comme Jade [FAB07], où les agents "véhicules" évoluent de manière dynamique et autonome dans leur environnement. Les SMA permettent à un agent d'avoir un "comportement " spécifique que l'on peut programmer, un agent peut également communiquer avec d'autres agents grâce à des communications ACL spécifiques aux SMA. Grâce à ces deux caractéristiques Jade nous permet de programmer nos agents véhicules à effectuer un routage par saut et à trouver le meilleur prochain agent relai à chaque itération. Dans un second temps nous allons tester et valider notre travail sous un simulateur de réseaux comme NS3, ou NS2 [GAJ13]. Les durées de contact résultant seront utilisées dans le protocole de routage géographique. Elles sont, en plus de la variable d'équilibrage de charge et du log d'anomalies de communication, décisives dans le choix d'une entité relai. En plus de l'injection des informations importantes à l'algorithme de routage, nous assurons également un système d'acquittement entre deux agents. Un véhicule $V_{1}$ reçoit $n$ paquets d'un véhicule émetteur $V_{0}$, lorsque $\mathrm{V}_{1}$ aura acheminé les $\mathrm{n}$ paquets à son propre relais $\mathrm{V}_{2}, \mathrm{~V}_{1}$ envoie un acquittement à $\mathrm{V}_{0}$ et attend l'acquittement de $\mathrm{V}_{2}$. Cet acquittement local entre deux nœuds contribue à la mise à jour des logs d'anomalies de communication et permet de vérifier la fiabilité d'un véhicule. Enfin pour rendre notre stratégie robuste et tolérante à la mobilité et aux problèmes de connectivité dans les réseaux à forte mobilité, nous ajoutons un acquittement global qui sera renvoyé à l'émetteur lorsque la totalité de la donnée arrive à la destination finale.

Comme perspective, nous allons élargir notre travail au-delà des communications unicast "1 à 1", aux communications de type MultiCast et BroadCast, afin de s'adapter à un plus large éventail d'application et de services IoV.

\section{Bibliographie}

[HUA13] J. M. Huang, «Research on Internet of Vehicles and its Application in Intelligent Transportation », Applied Mechanics and Materials, vol. 321-324, p. 2818-2821, 2013.

[REH13] S. ur Rehman, M. A. Khan, T. A. Zia, et L. Zheng, « Vehicular Ad-Hoc Networks (VANETs) - An Overview and Challenges », Journal of Wireless Networking and Communications, vol. 3, n 3, p. 29-38, 2013.

[FAN15] L. Fan, G. Li, J. Yang, H. Sun, et Z. Luo, « The design of IOV overall structure and the research of IOV key technology in intelligent transportation system », International Power, Electronics and Materials Engineering Conference, Dalian, Chine, 2015.

[IND14] A. Indra, R. Murali, et Venkatesh, «Routing Protocols for Vehicular Ad-hoc Networks (VANETs): A Review », Journal of Emerging Trends in Computing and Information Science, vol. 5, p. 25-43, 2014.

[GER16] M. Gerla, E. K. Lee, G. Pau, et U. Lee, «Internet of vehicles: From intelligent grid to autonomous cars and vehicular clouds », in 2014 IEEE World Forum on Internet of Things (WF-IoT), 2014, International Journal of Distributed Sensor Networks, Vol 12, p. 241-246, 2016.

[GHA15] N. Ghanishtha et J. Yogesh, «Review on classification of different VANET Protocols based on routing information », International Journal Of Advanced Reaserch in Computer and Communication Engineering, p. 388$392,2015$.

[MAO12] H. Maowad et E. Shaaban, « Enhancing AOMDV Routing Protocol for V2V Communication », in Proceedings of the 6th International Conference on Communications and Information Technology, and Proceedings of the 3rd World Conference on Education and Educational Technologies, Stevens Point, Wisconsin, USA, p. 20-27, 2012.

[CHEN15] J. Cheng, J. Cheng, M. Zhou, F. Liu, S. Gao, et C. Liu, « Routing in internet of vehicles: A review », IEEE Transactions on Intelligent Transportation Systems, vol. 16, $\mathrm{n}^{\circ}$ 5, p. 2339-2352, 2015. 
[KARP00] B. Karp et H.-T. Kung, "GPSR: Greedy perimeter stateless routing for wireless networks », Proceedings of the 6th annual international conference on Mobile computing and networking, Boston, MA, USA, p. 243-254, 2000.

[SHR12] K. Shrivastava et S. k Jain, «Enhanced Greedy Perimeter Stateless Routing Protocol (E-Gprs)», International Journal of Engineering Research and Technology, Vol.1-Issue 10, pp. 1-8, 2012.

[KAU16] S. Kaur et K. Kaur, « An New Improved GPSR (I-GPSR) Routing Protocol For VANET », Imperial Journal of Interdisciplinary Research, vol. 2, $\mathrm{n}^{\mathrm{0}}$ 7, pp. 1190-1196, 2016.

[NZO09] J. Nzouonta, N. Rajgure et G. Wang, et C. Borcea, «VANET Routing on City Roads Using Real-Time Vehicular Traffic Information », IEEE Transactions on Vehicular Technology, vol. 58, nº 7, p. 3609-3626, 2009.

[HAR14] S. Harrabi, W. Chainbi et K. Ghedira, «A multi-agent proactive routing protocol for Vehicular Ad-Hoc Networks ", The 2014 International Symposium on Networks, Computers and Communications (ISNCC 2014) Hammamet, Tunisia, p. 1-6, 2014.

[Reh15] W. Liang, Z. Li, H. Zhang, S. Wang, et R. Bie, « Vehicular Ad Hoc Networks: Architectures, Research Issues, Methodologies, Challenges, and Trends », International Journal of Distributed Sensor Networks, vol. 11, $\mathrm{n}^{\mathrm{o}}$ 8, $\mathrm{p}$. $745303,2015$.

[CHA12] O. Chakroun, S. Cherkaoui, et J. Rezgui, «MUDDS: Multi-metric Unicast Data Dissemination Scheme for 802.11p VANETs ", in 2012 8th International Wireless Communications and Mobile Computing Conference (IWCMC), Cyprus, p. 1074-1079, 2012.

[SHA11] K. Shafiee, A. Attar et V. C. M. Leung, «Optimal distributed vertical handoff strategies in vehicular heterogeneous networks », IEEE Journal on Selected Areas in Communications, Vol. 29, Issue. 3, p. 534 - 544, 2011.

[LAB12] H. Labiod, G el mouna Zhioua, N. Tabbane, et S. Tabbane, «An Efficient QoS Based Gateway Selection Algorithm for VANET to LTE Advanced Hybrid Cellular Network », in Proceedings of the 15th ACM International Conference on Modeling, Analysis and Simulation of Wireless and Mobile Systems, New York, NY, USA, p. 353$356,2012$.

[LI12] Y. Li, K. Ying, P. Cheng, H. Yu, et H. Luo, « Cooperative data dissemination in cellular-VANET heterogeneous wireless networks », in 2012 4th International High Speed Intelligent Communication Forum, Chine, p. 1- 4. 2012.

[ALS16] N. Alsharif, K. Aldubaikhy, et X. S. Shen, «Link duration estimation using neural networks based mobility prediction in vehicular networks », in Electrical and Computer Engineering (CCECE), 2016 IEEE Canadian Conference, Vancouver, BC, Canada, p. 1- 4, 2016.

[JER08] M. JERBI, « Protocoles pour les communications dans les réseaux de véhicules en environnement urbain : Routage et GeoCast basés sur les intersections », thèse de doctorat soutenue à université d'Evry Val d'Essonne, le 06 novembre 2008.

[ATE10] T. Atechian, «Protocole de routage géo-multipoint hybride et mécanisme d'acheminement de données pour les réseaux ad hoc de véhicules (VANETs) », thèse de doctorat soutenu à L'INSA Lyon, le 24 septembre 2010.

[GAJ13] K. Gajananan et al., "A cooperative its study on green light Optimisation using an integrated traffic, driving, and communication simulator », Proceedings 36th Australasian Transport Research Forum (ATRF), Brisbane, Queensland, Australia, 2013.

[ZHA08] J. Zhao, G. Cao, «VADD :Vehicle-assisted data delivery in vehicular ad hoc networks », IEEE Transaction on Vehicular Technology, Vol. 57, n³,p. 1910-1922, 2008.

[SUN06] W. Sun, H. Yamaguchi, K. Yukimasa., « A cooperative its study on green light Optimisation using an integrated traffic, driving, and communication simulator », Proceedings of 14th IEEE International Workshop on Quality of Service, IWQoS, New Haven, Connecticut, USA, 2006.

[FAB07] B. Fabio Luigi G. Caire, G. Dominic, « Developing multi-agent systems with JADE », John Wiley \& Sons Publisher, Vol.7, 2007. 